54 No. 1/2007, 167-174

on-line at: www.actabp.pl

Regular paper

\title{
The involvement of protein kinase $A$ in the immune response of Galleria mellonella larvae to bacteria
}

\author{
Małgorzata Cytryńska, Agnieszka Zdybicka-Barabas and Teresa Jakubowicz ${ }^{\bowtie}$ \\ Department of Invertebrate Immunology, Institute of Biology, Maria Curie-Skłodowska University, \\ Lublin, Poland
}

Received: 14 September, 2006; revised: 11 December, 2006; accepted: 17 January, 2007 available on-line: 20 February, 2007

\begin{abstract}
The role of protein kinase A (PKA) in the humoral immune response of the greater wax moth Galleria mellonella larvae to live Gram-positive bacteria Micrococcus lysodeikticus and Gramnegative bacteria Escherichia coli was investigated. The immune challenge of larvae with both kinds of bacteria caused an increase in fat body PKA activity depending on the injected bacteria. Gram-positive $M$. lysodeikticus was a much better inducer of the enzyme activity than Gram-negative $E$. coli. The PKA activity was increased about 2.5 -fold and 1.5 -fold, after M. lysodeikticus and $E$. coli injection, respectively. The in vivo inhibition of the enzyme activity by a cell permeable selective PKA inhibitor, Rp-8-Br-cAMPS, was correlated with considerable changes of fat body lysozyme content and hemolymph antimicrobial activity in bacteria-challenged insects. The kinetics of changes were different and dependent on the bacteria used for the immune challenge of G. mellonella larvae.
\end{abstract}

Keywords: Galleria mellonella, protein kinase A, lysozyme, antibacterial activity, Rp-8-Br-cAMPS

\section{INTRODUCTION}

The characteristic feature of invertebrate immunity is the lack of an adaptive immune response. In insects, pathogen recognition leads to activation of humoral and cellular innate defence mechanisms. The cellular response comprises phagocytosis, encapsulation and nodulation of non-self bodies. In the humoral response, a crucial role is played by antimicrobial peptides, synthesized in the fat body (a functional analog of mammalian liver) and hemocytes after non-self recognition and then released into the hemolymph. Literature data indicated the involvement of cAMP-dependent protein kinase (protein kinase $\mathrm{A}, \mathrm{PKA}$ ) in the regulation of the immune response in insects. Brooks and Dunphy (2005) demonstrated that active PKA limited the greater wax moth Galleria mellonella hemocyte response against Xenorhabdus nematophila and Bacillus subtilis in vitro and in vivo. PKA inhibition by a selective inhibitor, Rp-8-Br-cAMPS (Rp-isomer of 8-bromoadenosine $3^{\prime}, 5^{\prime}$-cyclic monophosphorothioate), increased the number of hemocytes with adherent bacterial cells and enhanced phagocytosis of bacteria in vitro as well as the removal of bacteria from hemolymph in vivo (Brooks \& Dunphy, 2005). In contrast, an elevation of cellular cAMP concentration led to an impaired response of G. mellonella hemocytes to both kinds of bacteria (Brooks \& Dunphy, 2005; Marin et al., 2005). Moreover, the inhibition of PKA by another inhibitor, H89 ( $\mathrm{N}$-[2-( $p$-bromocinnamylamino)ethyl]-5-iso-quinolinesulfonamide), increased the adhesion of G. mellonella granulocytes to glass slides, indicating PKA involvement in the regulation of hemocyte adhesion properties (Zakarian et al., 2003).

PKA was implicated in the regulation of antimicrobial peptide synthesis in insects. It was suggested that PKA is one of the necessary factors for

${ }^{\square}$ Corresponding author: Teresa Jakubowicz, Department of Invertebrate Immunology, Institute of Biology, Maria CurieSkłodowska University, Akademicka 19, 20-033 Lublin, Poland; tel.: (48 81) 537 5089; e-mail: tejak@biotop.umcs.lublin.pl Abbreviations: cfu, colony forming units; EWL, egg white lysozyme; H89, N-[2-(p-bromocinnamylamino)ethyl]-5-isoquinolinesulfonamide; LPS, lipopolysaccharide; PG, peptidoglycan; PKA, protein kinase A; PMSF, phenylmethylsulfonyl fluoride; PTU, phenylthiourea; Rp-8-Br-cAMPS, Rp-isomer of 8-bromoadenosine 3',5'-cyclic monophosphorothioate. 
the activation of cecropin B genes in isolated Bombyx mori hemocytes (Taniai et al., 1996). The expression of cecropin B gene was triggered by dibutyrylcAMP, a cell-permeable cAMP analog (Shimabukuro et al., 1996). In addition, the expression of that gene induced by LPS or lipid A was inhibited by H89, a PKA inhibitor (Shimabukuro et al., 1996; Taniai et al., 1996).

In Drosophila melanogaster at least two signalling pathways, Toll and Imd, are involved in the regulation of antimicrobial peptide gene expression (Leclerc \& Reichhart, 2004; Iwanaga \& Lee, 2005; Tanji \& Ip, 2005; Pinheiro \& Ellar, 2006; Wang \& Ligoxygakis, 2006). The activation of Toll and Imd pathways leads to the release of $\mathrm{NF \kappa B} / \mathrm{Rel}$ family transcription factors Dif, Dorsal, Relish. Nuclear transport and activation of D. melanogaster factor Dorsal was dependent on phosphorylation by PKA (Norris \& Manley, 1992) and it was shown that the phosphorylation of Dorsal by PKA at Ser312 facilitated an interaction of Dorsal molecule with importin (Briggs et al., 1998).

In our recent paper, we demonstrated PKA activity in the fat body of G. mellonella larvae (Cytryńska et al., 2006). The enzyme activity was considerably increased after the immune challenge of animals with Escherichia coli LPS. The inhibition of PKA by H89 or Rp-8-Br-cAMPS in vivo was correlated with a lower level of antimicrobial activity in the hemolymph of immune-challenged insects. A considerable decrease in the content of lysozyme, which plays an important role in the lepidopteran insect immune response, was also detected in the fat body of inhibitor pre-treated LPS-challenged larvae. Our results demonstrated the involvement of PKA in G. mellonella humoral immune response to LPS, a cell wall component of Gram-negative bacteria.

In this paper, we investigated a possible role of PKA in the humoral immune response of G. mellonella larvae to live Gram-negative bacteria E. coli and Gram-positive bacteria Micrococcus lysodeikticus. We used a cell permeable, selective PKA inhibitor, Rp-8-Br-cAMPS, and examined the effect of PKA inhibition in vivo on hemolymph antimicrobial activity and lysozyme content in the fat body of bacteriachallenged insects.

\section{MATERIALS AND METHODS}

Insect culture, immune challenge and hemolymph collection. Larvae of the greater wax moth Galleria mellonella (Lepidoptera: Pyralidae) were reared on a natural diet - honeybee nest debris at $30^{\circ} \mathrm{C}$ in the dark. Last instar larvae (250-300 $\mathrm{mg}$ in weight) were used throughout the study.
For PKA activity inhibition in vivo, the larvae were injected with $1.5 \mathrm{nmol}$ of Rp-8-Br-cAMPS (Sig$\mathrm{ma}$ ) in $3 \mu \mathrm{l}$ of apyrogenic water (the approximate concentration of the inhibitor in larval hemolymph $-20 \mu \mathrm{M})$. Control animals were injected with the same volume of apyrogenic water. In some experiments, 15 min after inhibitor administration, the larvae were immune-challenged by injection of $3 \mu$ of apyrogenic water containing live E. coli D31 $\left(10^{5} \mathrm{cfu}\right)$ or M. lysodeikticus $\left(5 \times 10^{4} \mathrm{cfu}\right)$. After the treatment, the larvae were kept at $30^{\circ} \mathrm{C}$ in the dark on sterile Petri dishes and the hemolymph was collected after the time indicated in the text.

The hemolymph collection was performed into sterile and chilled Eppendorf tubes containing a few crystals of phenylthiourea to prevent melanisation, as described earlier (Cytryńska et al., 2006). The hemocyte-free hemolymph was obtained by centrifugation at $200 \times g$ for $5 \mathrm{~min}$ to pellet hemocytes and the supernatant was subsequently centrifuged at $20000 \times \mathrm{g}$ for $15 \mathrm{~min}$ at $4^{\circ} \mathrm{C}$ to pellet cell debris. The cell-free hemolymph was used immediately for testing antibacterial activity.

Antibacterial activity assay. For antibacterial activity tests, the LPS defective, streptomycin and ampicillin resistant mutant of E. coli K12, strain D31 was used (Boman et al., 1974). The antibacterial activity in the hemolymph was detected by a welldiffusion method using solid agar plates containing viable E. coli cells (Hoffmann et al., 1981) and hen egg white lysozyme (EWL) at a concentration of 2.0 $\mathrm{mg} \times \mathrm{ml}^{-1}$ to improve the method sensitivity (Chalk \& Suliaman, 1998; Cytryńska et al., 2001). Each well on the Petri dish was filled with $4 \mu \mathrm{l}$ of hemolymph diluted four times with sterile water. After incubation of the agar plates at $37^{\circ} \mathrm{C}$ for $24 \mathrm{~h}$ the diameters of E. coli D31 growth inhibition zones were measured and the level of antimicrobial activity was calculated using the algorithm described by Hultmark et al. (1982). For evaluation of antibacterial activity, synthetic cecropin B (Sigma) was used as a standard.

Preparation of hemolymph extracts. Acidic/ methanolic extracts of hemocyte-free hemolymph were obtained by the method adapted from Schoofs et al. (1990). The hemolymph was diluted ten times with the extraction solution consisting of methanol/ glacial acetic acid/water (90:1:9, by vol.) and mixed thoroughly. Precipitated proteins were pelleted by centrifugation at $20000 \times \mathrm{g}$ for $30 \mathrm{~min}$ at $4^{\circ} \mathrm{C}$. The obtained supernatant, containing peptides and proteins of molecular mass below $30 \mathrm{kDa}$, was collected, freeze-dried and the pellet was stored at $-20^{\circ} \mathrm{C}$ until needed. For SDS/PAGE, the pellet was dissolved in an appropriate volume of sample buffer according to Schägger and von Jagow (1987). 
Isolation of fat bodies and preparation of cell-free extracts. For fat body isolation, the larvae were anaesthesized by submerging in ice-cold apyrogenic water and then surface disinfected with $70 \%$ ethanol. The fat bodies were dissected under sterile ice-cold physiological saline $(172 \mathrm{mM} \mathrm{KCl}$, $68 \mathrm{mM} \mathrm{NaCl}, 5 \mathrm{mM} \mathrm{NaHCO}{ }_{3}$ pH 6.1, osmolarity 420 mOsm) (Vilcinskas \& Matha, 1997). After dissection, the fat body was transferred into a sterile, chilled Eppendorf tube containing $1 \mathrm{ml}$ of physiological saline. Then the solution was removed and the fat body was frozen in liquid nitrogen.

Cell-free extracts of fat bodies were prepared in ice-cold PKA buffer $(50 \mathrm{mM}$ Tris/ $\mathrm{HCl}, \mathrm{pH}$ 7.4, $10 \mathrm{mM} \beta$-glycerophosphate, $2.5 \mathrm{mM}$ sodium pyrophosphate, $0.5 \mathrm{mM}$ EDTA, $1 \mathrm{mM}$ phenylmethylsulfonyl fluoride (PMSF), $6 \mathrm{mM} \beta$-mercaptoethanol), as described earlier (Cytryńska et al., 2006). The obtained extracts were centrifuged at $20000 \times g$ for $15 \mathrm{~min}$ at $4^{\circ} \mathrm{C}$, the supernatants were collected and were used immediately for PKA activity assay. For immunoblotting, an appropriate volume of Laemmli sample buffer (Laemmli, 1970) was added and the samples were stored at $-20^{\circ} \mathrm{C}$.

Protein kinase A activity assay. PKA activity was measured by a non-radioactive method using PepTag ${ }^{\circledR}$ Assay (Promega), as described (Cytryńska et al., 2006). Briefly, the reaction mixture in the volume of $25 \mu \mathrm{l}$ contained: $20 \mathrm{mM}$ Tris/ $\mathrm{HCl}, \mathrm{pH}$ 7.4, $10 \mathrm{mM} \mathrm{MgCl}, 1 \mathrm{mM}$ ATP, $1 \mu \mathrm{M}$ cAMP, $2.5 \mathrm{mM}$ sodium pyrophosphate, $2.5 \mathrm{mM} \quad \beta$-glycerophosphate, $2 \mu \mathrm{g}$ of PepTag ${ }^{\circledR}$ A1 Peptide (specific PKA substrate) and $20 \mu \mathrm{g}$ of fat body total proteins. Each reaction was performed at room temperature for $30 \mathrm{~min}$ and then stopped by heating the incubation mixture at $95^{\circ} \mathrm{C}$ for $10 \mathrm{~min}$. PepTag ${ }^{\circledR}$ A1 Peptide fluorescence was visualized after $20 \mathrm{~min}$ of horizontal electrophoresis in $0.8 \%$ agarose by using a UV transilluminator.

Immunoblotting. Samples of fat body extracts $(80 \mu \mathrm{g}$ of total protein) were subjected to $13.8 \%$ glycine SDS/PAGE and electroblotted onto Immobilon membranes (Millipore) for $90 \mathrm{~min}$ at $350 \mathrm{~mA}$. For lysozyme identification, the membranes were probed with polyclonal antibodies (1:1000) to G. mellonella lysozyme, a generous gift of Prof. I. H. Lee (Department of Life Science, Hoseo University, South Korea). As second antibodies, alkaline phosphatase-conjugated goat anti-rabbit IgGs (1:30000) were used and immunoreactive bands were visualized by incubation with $p$-Nitroblue Tetrazolium chloride and 5-bromo-4-chloro-3-indolyl phosphate.

Other methods. The protein concentration was estimated by the Bradford method using bovine serum albumin (BSA) as a standard (Bradford, 1976).
Polyacrylamide gel electrophoresis of proteins was performed by $13.8 \%$ glycine SDS/PAGE according to Laemmli (1970). Low molecular mass proteins and peptides were resolved by $16.5 \%$ tricine SDS/ PAGE according to Schägger and von Jagow (1987).

The densitometric analysis of bands was performed using Quantity One computer imaging system (BioRad, Hercules, CA, USA). All data are presented as means \pm S.D. for at least three experiments.

In order to compare two means, statistical analysis was performed by Student's $t$-test. Significance was established at $P<0.001$ (Figs. $1,2,3$ ) or $P<0.01$ (Fig. 4).

\section{RESULTS}

PKA activity in the fat body of bacteria-challenged G. mellonella larvae

In the present work, we investigated the level of PKA activity in the fat body of bacteria-challenged insects. In general, bacterial challenge caused an increase in PKA activity but the kinetics and the level of the enzyme activity differed considerably depending on the injected bacteria. In the larvae challenged with Gram-positive bacteria M. lysodeikticus, a considerable increase in the enzyme activity was noticed when compared to the water-treated larvae (Fig. 1). About a 2.5-fold increase in PKA activity was detected as early as $15 \mathrm{~min}$ after the challenge and a high level of enzyme activity was sustained until $24 \mathrm{~h}$ after injection of bacteria (Fig. 1).

When G. mellonella larvae were immune-challenged with Gram-negative bacteria E. coli, a different kinetics of fat body PKA activity was observed (Fig. 1). In general, the enzyme activity level in E. coli-challenged larvae was lower in comparison to the level measured in M. lysodeikticus-injected insects. In most time points after the challenge, PKA activity oscillated around the naïve larvae level, however, starting from about $15 \mathrm{~min}$ after the treatment, it was increased when compared to water-treated animals. A slight, nevertheless significant $(P<0.001)$ increase in the enzyme activity was detected $45 \mathrm{~min}$ after bacteria injection. A more considerable increase in PKA activity, 1.5-fold and 1.6-1.8-fold, respectively, was detected 2 and then 6-24 h after the challenge, in comparison to the measurements in water-treated larvae. However, a transient decrease by $29.6 \%$ of PKA activity was detected $4 \mathrm{~h}$ after treatment with E. coli (Fig. 1).

When G. mellonella larvae, before bacteria injection, were pre-treated with PKA inhibitor, Rp8-Br-cAMPS, a fall in PKA activity was noticed 


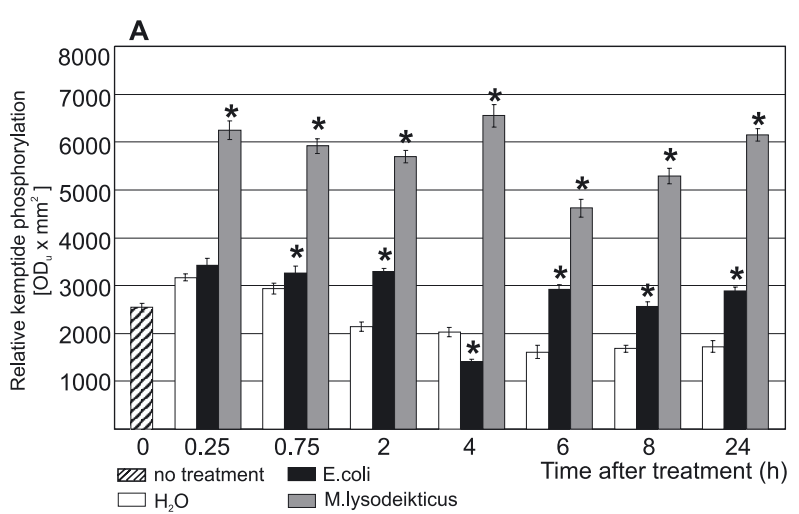

B

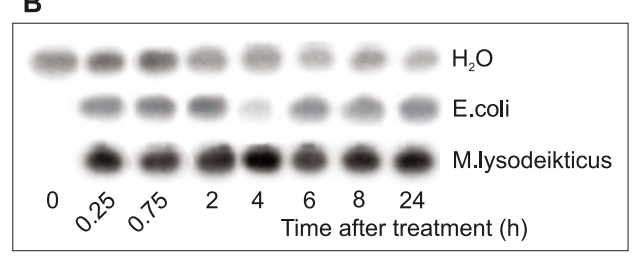

Figure 1. PKA activity in the fat body of bacteria-challenged G. mellonella larvae.

The larvae (five individuals per each group) were injected with water, $M$. lysodeikticus or E. coli suspension as described in Materials and Methods. The fat bodies were isolated at the indicated time points, cell-free extracts were prepared and PKA activity was measured. Diagram (A) presents the densitometric analysis of bands containing phosphorylated kemptide and photo (B) shows corresponding fragments of the agarose gels. The first hatched bar represents PKA activity in the fat body of non-treated larvae. *Statistically significant differences $(P<0.001)$ from samples after $\mathrm{H}_{2} \mathrm{O}$ treatment at respective time points.

(Fig. 2). Fifteen minutes after the challenge with M. lysodeikticus, the PKA activity was decreased by $7.4 \%$. A more evident impairment of PKA activity, by $27.5-67.3 \%$, was detected $4-24 \mathrm{~h}$ after $M$. lysodeikticus injection (Fig. 2A). When PKA inhibitor was used before E. coli injection, $15 \mathrm{~min}$ after the challenge PKA activity in the fat body was lowered by $10.6 \%$ and then, $2-24 \mathrm{~h}$ after the challenge, by $25-$ $52.3 \%$ (Fig. 2B).

Lysozyme content in the fat body of bacteria-challenged larvae, pre-treated with PKA inhibitor

Literature data indicate that lysozyme plays an important role in insect innate immunity (Powning \& Davidson, 1973; Chadwick \& Aston, 1991; Hultmark, 1996). The protein is synthesized mainly in the fat body and hemocytes and released into the hemolymph. The constitutive, low level of lysozyme is present in lepidopteran insect hemolymph, however, non-self recognition leads to an increase in the hemolymph lysozyme content and activity (Sun et al., 1991; Morishima et al., 1995; Chung \& Ourth, 2000; Lavine et al., 2005). Recently, we demonstrated that PKA activity inhibition was correlated
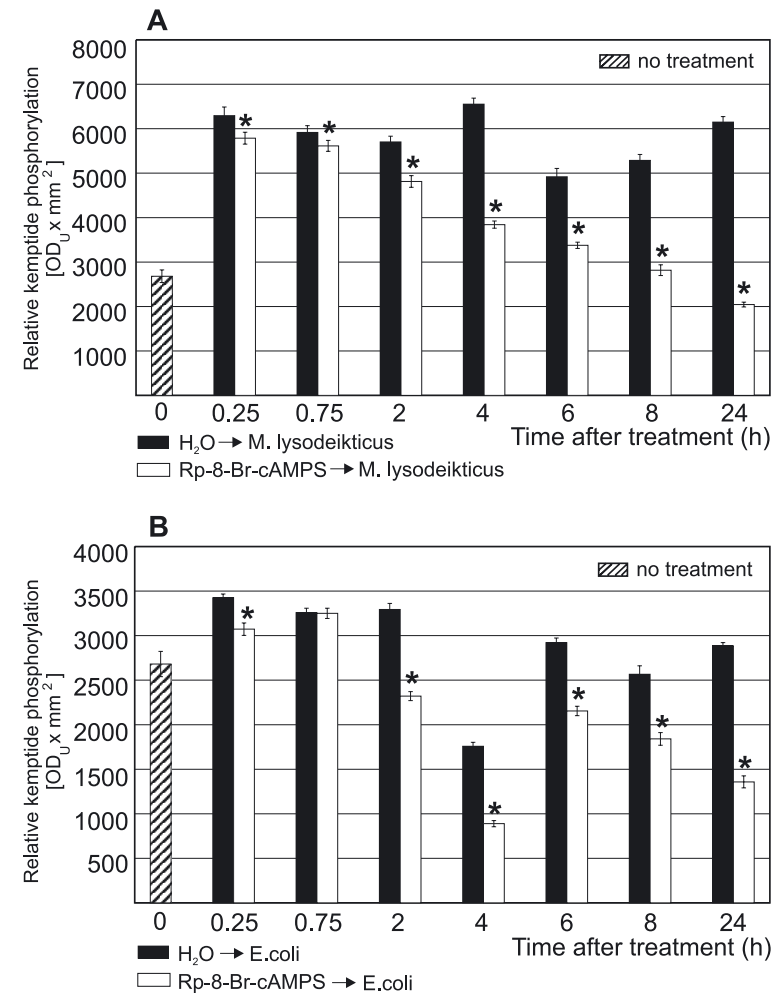

Figure 2. PKA activity in the fat body of G. mellonella larvae challenged with M. lysodeikticus (A) and E. coli (B) pre-treated with PKA inhibitor.

The larvae (five individuals per each group) were injected with Rp-8-Br-cAMPS or water and then with suspension of $M$. lysodeikticus or E. coli. The fat bodies were isolated at the indicated time points and PKA activity was measured as described in Materials and Methods. The first hatched bars represent PKA activity in fat body of non-treated larvae. *Statistically significant differences $(P<0.001)$ Rp8-Br-cAMPS versus no Rp-8-Br-cAMPS at respective time points.

with a considerable decrease in lysozyme content in fat body of LPS-challenged larvae (Cytryńska et al., 2006).

In the present study, we investigated whether similar correlation occurred between PKA activity inhibition and lysozyme content in the fat body of $M$. lysodeikticus- and E. coli-challenged larvae (Fig. 3). In the fat body of bacteria-challenged larvae, lysozyme was detected 4 and $6 \mathrm{~h}$ after treatment with $M$. lysodeikticus and E. coli, respectively, although at a relatively low level (Fig. 3). Then the lysozyme content gradually increased reaching the highest level $24 \mathrm{~h}$ after the challenge (Fig. 3A, B). When bacteria-injected insects were pre-treated with PKA inhibitor, lysozyme in the fat body was detected earlier than in water pre-treated insects, which means $2 \mathrm{~h}$ after injection of bacteria (Fig. 3A, B). In Rp-8-Br-cAMPS pre-treated M. lysodeikticus-challenged larvae, $2 \mathrm{~h}$ after the challenge, a high level of the protein was detected (Fig. 3A). The densitometric analysis revealed that it reached $82.4 \%$ of the lysozyme content measured in the fat body of water pre-treated lar- 
A

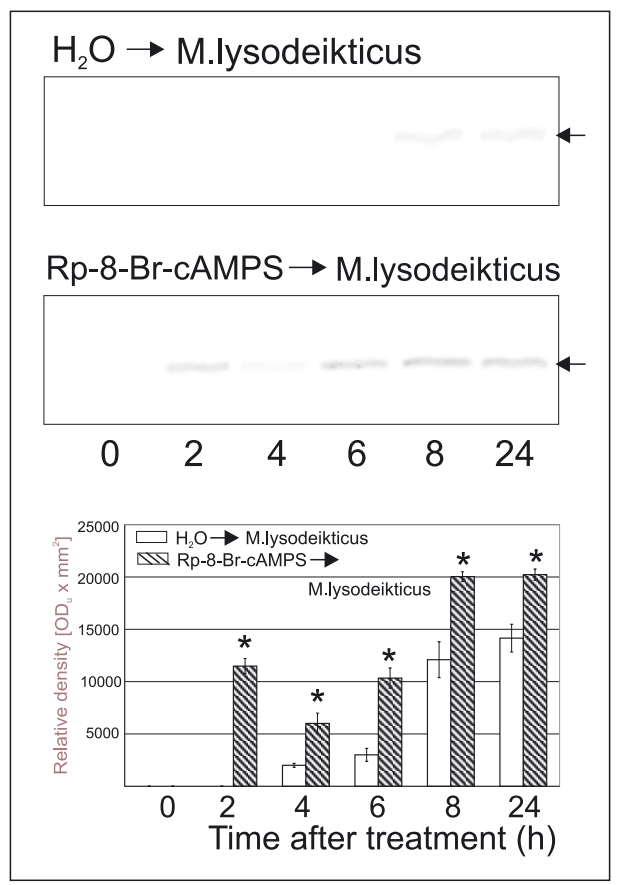

B

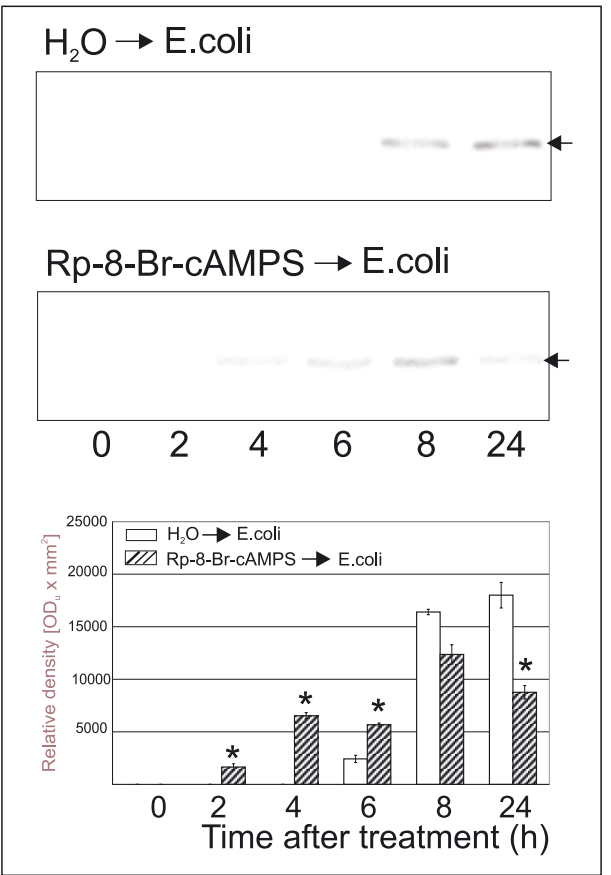

Figure 3. Detection of lysozyme in the fat body of G. mellonella larvae challenged with M. lysodeikticus (A) and E. coli (B) pre-treated with PKA inhibitor.

The larvae (five individuals per each group) were injected with Rp-8-Br-cAMPS or water and then with suspension of M. lysodeikticus or E. coli. The fat bodies were isolated at the indicated time points and prepared for immunoblotting. The samples were resolved by glycine SDS/PAGE and transferred onto Immobilon membranes. The membranes were probed with antibodies against G. mellonella lysozyme. The lysozyme band is marked by an arrow. The diagrams represent the densitometric analysis of bands containing lysozyme. ${ }^{*}$ Statistically significant differences $(P<0.001) \mathrm{Rp}-8$ - $\mathrm{Br}$ cAMPS versus no Rp-8-Br-cAMPS at respective time points.

vae $24 \mathrm{~h}$ after challenge. A further increase in lysozyme content, by 4-, 1.6-, and 1.5-fold, respectively, was noticed 6,8 and $24 \mathrm{~h}$ after injection of bacteria. However, $4 \mathrm{~h}$ after the challenge in Rp-8-Br-cAMPS pre-treated animals, a transient decrease in lysozyme content was noticed (Fig. 3A).

Different results were obtained when lysozyme content was examined in E. coli-challenged larvae pre-treated with PKA inhibitor (Fig. 3B). Starting from about $8 \mathrm{~h}$ after $E$. coli injection, the lysozyme content in the fat body of these animals was lowered and $24 \mathrm{~h}$ after the challenge it reached $50 \%$ of that measured in water pre-treated larvae (Fig. 3B). The results obtained for E. coli-challenged larvae resembled, to some extent, those presented for LPS-injected insects pre-treated with PKA inhibitor, where a diminishing in fat body lysozyme content was also detected (Cytryńska et al., 2006).

Antibacterial activity in the hemolymph of bacteriachallenged larvae, pre-treated with PKA inhibitor

It is well documented that bacteria infection induces the synthesis of antimicrobial peptides in the insect fat body which are secreted into the hemolymph of challenged larvae (Dickinson et al., 1988; Yamakawa \& Tanaka, 1999; Lowenberger, 2001; Mak et al., 2001; Hultmark, 2003; Royet et al., 2005). We investigated the level of antibacterial activity in the hemolymph of bacteria-challenged larvae pre-treated with PKA inhibitor.

Immune challenge of G. mellonella larvae with M. lysodeikticus or E. coli caused the appearance of antibacterial activity in the hemolymph (Fig. 4). The activity was detected as early as $2 \mathrm{~h}$ after the challenge and it gradually increased reaching, $24 \mathrm{~h}$ after injection of bacteria, the level equivalent to cecropin B activity of $6 \mu \mathrm{M}$ and $4.5 \mu \mathrm{M}$, for M. lysodeikticusand E. coli-challenged larvae, respectively (Fig. 4A, B). The induction of antibacterial activity was correlated with the appearance in the hemolymph of additional peptides of molecular mass below $6.5 \mathrm{kDa}$ (Fig. 4C).

When M. lysodeikticus-challenged larvae were pre-treated with PKA inhibitor, the antibacterial activity level measured 3-4 h after the challenge was diminished by $33.3 \%$ and $28.6 \%$, respectively, in comparison to water pre-treated animals. In contrast, an increase in antibacterial activity, reaching 1.4-fold $24 \mathrm{~h}$ after treatment, was detected 8-24 h after the challenge (Fig. 4A).

Pre-treatment of G. mellonella larvae with $\mathrm{Rp}$ 8-Br-cAMPS before E. coli injection resulted in a lower level of hemolymph antibacterial activity than 

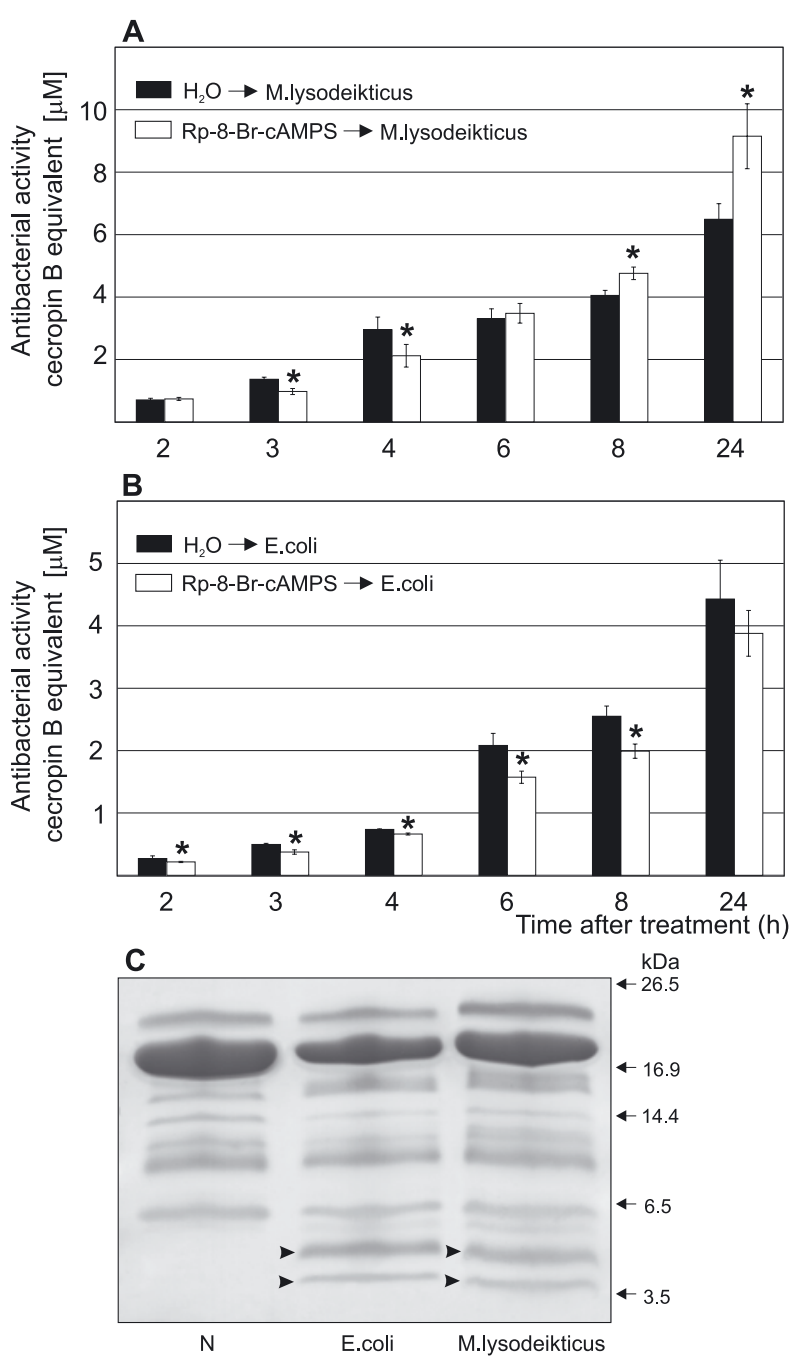

Figure 4. Antibacterial activity in the hemolymph of $G$. mellonella larvae challenged with M. lysodeikticus (A) and E. coli (B) pre-treated with PKA inhibitor.

The larvae (ten individuals per each group) were injected with Rp-8-Br-cAMPS or water and then with suspension of M. lysodeikticus or E. coli. The hemolymph was collected at the indicated time points and used for antimicrobial activity assay as described in Materials and Methods. The photo (C) presents tricine SDS/PAGE analysis of extracts prepared from hemolymph obtained $24 \mathrm{~h}$ after bacteria injection and from naive larvae hemolymph (N). Additional peptide bands present in hemolymph of bacteria-challenged insects are marked by arrowheads. *Statistically significant differences $(P<0.01) \mathrm{Rp}-8$-Br-cAMPS versus no $\mathrm{Rp}-8$-Br-cAMPS at respective time points.

in water pre-treated animals (Fig. 4B). In particular, 6 and $8 \mathrm{~h}$ after the treatment it reached $74.1 \%$ and $78 \%$, respectively, of that measured in the hemolymph of water pre-treated insects (Fig. 4B).

\section{DISCUSSION}

We showed recently that the immune challenge of G. mellonella larvae with E. coli LPS led to a considerable, about 2-fold, increase in fat body PKA activity (Cytryńska et al., 2006). Here we demonstrated that infection of G. mellonella larvae with live Gram-positive and Gram-negative bacteria influenced the level of PKA activity depending on the bacteria used for immune challenge. After bacterial treatment, the increased level of PKA activity was sustained for $24 \mathrm{~h}$, similarly to the results obtained for LPS-challenged insects (Cytryńska et al., 2006). However, Gram-positive M. lysodeikticus appeared to be a much better inducer of PKA activity than Gram-negative E. coli. Although E. coli cells (containing LPS) were used for G. mellonella challenge in a 2-fold higher amount than M. lysodeikticus, PKA activity was induced to a much lower level. It is known that the bacterial cell wall composition is an important determinant of insect immune response induction. Studies performed on D. melanogaster indicated that especially the type of peptidoglycan (PG) molecules determined the activation of certain signalling pathways involved in innate immunity mechanisms (Leulier et al., 2003; Kurata, 2004; Kurata et al., 2006). Most Gram-positive bacteria, whose cell walls contain a thick layer of lysine type PG, activate the Toll pathway. The Gram-negative bacteria, containing cell walls composed of a thin layer of diaminopimelic type PG covered by LPS, activate the Imd signalling pathway. The infection of D. melanogaster with different classes of bacteria induces preferential synthesis of different repertoires of defence peptides in the fat body (Leclerc \& Reichhart, 2004; Tanji \& Ip, 2005; Pinheiro \& Ellar, 2006; Wang \& Ligoxygakis, 2006). Although signalling pathways activated in G. mellonella larvae after bacterial infection are not known at present, it was demonstrated that PKA (Cytryńska et al., 2006) and JNK MAP kinase (Wojda et al., 2004) are engaged in the humoral immune response in LPS-challenged G. mellonella larvae. The differences in the level and the kinetics of PKA activation after G. mellonella challenge with Gram-positive and Gram-negative bacteria indicate the functioning of different signalling pathways responding to both classes of bacteria in which PKA plays a regulatory role.

The use of a cell permeable selective PKA inhibitor, Rp-8-Br-cAMPS, before injection of bacteria, led to a decrease in fat body PKA activity in $M$. lysodeikticus- as well as E. coli-challenged larvae. The kinetics of PKA activity changes in Rp-8-Br-cAMPS pre-treated animals might suggest that the enzyme inhibition detected shortly after the challenge evoked further changes in cellular as well as in the whole organism metabolism, which in turn influenced the PKA activity later after the challenge.

The inhibition of PKA activity considerably coincided with changes in the fat body lysozyme content. In the fat body of larvae pre-treated with 
PKA inhibitor, lysozyme appeared at least $2 \mathrm{~h}$ earlier than in the water pre-treated ones. This seems to indicate that active PKA may to some extent regulate the process of lysozyme synthesis and/or promote a release of this protein from the fat body to the hemolymph in bacteria-challenged larvae. Our data might suggest that PKA, at least within the first hours after the challenge, is involved in negative regulation of lysozyme content in the fat body of bacteria-injected G. mellonella larvae. It is consistent with the results of studies on G. mellonella cellular immune response, where active PKA limited hemocyte reactions against $X$. nematophila and $B$. subtilis and inhibition of the enzyme enhanced phagocytosis of bacteria and increased adhesion properties of granulocytes (Zakarian et al., 2003; Brooks \& Dunphy, 2005; Marin et al., 2005).

Interestingly, changes in fat body lysozyme content under the conditions of PKA inhibition were dependent on the bacteria used for the immune challenge. A considerable increase in the lysozyme content was detected in the fat body of M. lysodeikticuschallenged insects. After E. coli injection, a decrease in the protein level was observed, resembling the results obtained for LPS-challenged larvae (Cytryńska et al., 2006). This probably reflects differences in immune reaction of G. mellonella larvae to both bacteria and also indicates PKA participation in the modulation of these processes.

PKA inhibition also influenced the hemolymph antibacterial activity. The kinetics of changes in the antibacterial activity level in Rp-8-Br-cAMPS pre-treated animals was dependent on the injected bacteria. However, the temporary decrease in the studied activity detected in hemolymph of larvae challenged with both bacteria could suggest the enzyme participation in the regulation of antimicrobial peptides synthesis and/or their release from fat body cells. So far, the engagement of PKA in the regulation of the cecropin $\mathrm{B}$ gene in $B$. mori hemocytes has been described (Taniai et al., 1996; Shimabukuro et al., 1996). The differences in antibacterial activity level in hemolymph of M. lysodeikticus- and E. colichallenged larvae could be connected with a higher and a lower content of lysozyme released from the fat body, respectively.

It was shown that the low constitutive level of lysozyme present in the hemolymph of lepidopteran insect non-immune larvae plays a significant role in peptidoglycan digestion. Soluble PG fragments released by lysozyme from bacterial cell walls can act as signalling molecules for activation of antimicrobial peptide synthesis, including lysozyme (Dunn et al., 1985; Kanost et al., 1988). Concerning the differences in PG content in cell walls of Grampositive and Gram-negative bacteria, its accessibility for and susceptibility to lysozyme, one can speculate that considerably more soluble PG fragments might be released from $M$. lysodeikticus than from E. coli cell walls. Inhibition of PKA activity together with the presence of different type and amount of PG in insects challenged with these bacteria could result in the demonstrated changes in fat body lysozyme content and hemolymph antibacterial activity level depending on bacteria used for immune challenge.

It should be noted that pre-treatment of $E$. coli- but not $M$. lysodeikticus-challenged larvae with PKA inhibitor caused changes similar, to some extent, to those detected in LPS-injected larvae pretreated with PKA inhibitors (Cytryńska et al., 2006). The observed similarities could be a result of the LPS presence in E. coli cell walls and the differences probably reflect a more complex reaction of G. mellonella larvae to whole bacterial cells.

The presented results suggest the involvement of PKA in the humoral immune response of G. mellonella larvae to Gram-positive as well as Gram-negative bacteria. We demonstrated that injection of bacteria caused an increase in PKA activity in the fat body of G. mellonella larvae. The inhibition of the enzyme activity was correlated with changes in the fat body lysozyme content and antibacterial activity level in the hemolymph of bacteria-challenged insects. The observed changes were dependent on the bacteria used for immune challenge. This might suggest the participation of different pathways responding to both classes of bacteria in G. mellonella larvae. Whether it results in the appearance of a different set of antimicrobial peptides remains to be elucidated.

\section{Acknowledgements}

This work was supported by the Ministry of Science and Higher Education, Poland (grant No. 2 P04C 080 29).

\section{REFERENCES}

Boman HG, Nilsson-Faye I, Paul K, Rasmuson T Jr (1974) Insect immunity. 1. Characteristics of an inducible cellfree antibacterial reaction in hemolymph of Samia cynthia pupae. Infect Immun 10: 136-145.

Bradford MM (1976) A rapid and sensitive method for the quantitation of microgram quantities of protein utilizing the principle of protein-dye binding. Anal Biochem 72: $248-254$.

Briggs LJ, Stein D, Goltz J, Corrigan VC, Efthymiadis A, Hübner S, Jans DA (1998) The cAMP-dependent protein kinase site $\left(\mathrm{Ser}^{312}\right.$ ) enhances Dorsal nuclear import through facilitating nuclear localization sequence/importin interaction. J Biol Chem 273: 22745-22752.

Brooks CL, Dunphy GB (2005) Protein kinase A affects Galleria mellonella (Insecta: Lepidoptera) larval haemocyte non-self responses. Immunol Cell Biol 83: 150-159.

Chadwick JS, Aston WP (1991) Antibacterial immunity in Lepidoptera. In Immunology of insects and other arthro- 
pods. Gupta AP, ed, pp 347-370. CRC Press, Boca Raton, Ann Arbor, London.

Chalk R, Suliaman WY (1998) Antimicrobial peptides from small insects: methods for insect culture and for the detection, visualization, isolation and sequencing of active hemolymph peptides. In Techniques in insect immunology. Wiesner A, Dunphy GB, Marmaras VJ, Morishima I, Sugumaran M, Yamakawa M, eds, pp 109-124. SOS Publications, USA.

Chung KT, Ourth DD (2000) Larval and pupal induction and N-terminal amino acid sequence of lysozyme from Heliothis virescens. J Insect Physiol 46: 563-572.

Cytryńska M, Zdybicka-Barabas A, Jabłoński P, Jakubowicz T (2001) Detection of antibacterial polypeptide activity in situ after sodium dodecyl sulfate-polyacrylamide gel electrophoresis. Anal Biochem 299: 274-276.

Cytryńska M, Zdybicka-Barabas A, Jakubowicz T (2006) Studies on the role of protein kinase A in humoral immune response of Galleria mellonella larvae. J Insect Physiol 52: 744-753.

Dickinson L, Russell V, Dunn PE (1988) A family of bacteria-regulated, cecropin D-like peptides from Manduca sexta. J Biol Chem 263: 19424-19429.

Dunn PE, Dai W, Kanost MR, Geng CX (1985) Soluble peptidoglycan fragments stimulate antibacterial protein synthesis by fat body from larvae of Manduca sexta. Dev Comp Immunol 9: 559-568.

Hoffmann D, Hultmark D, Boman HG (1981) Insect immunity: Galleria mellonella and other Lepidoptera have cecropia-P9-like factors active against Gram negative bacteria. Insect Biochem 11: 537-548.

Hultmark D (1996) Insect lysozymes. In Lysozymes: model enzymes in biochemistry and biology. Jollés P, ed, pp 87102. Birkhäuser Verlag Basel/Switzerland.

Hultmark D (2003) Drosophila immunity: paths and patterns. Curr Opin Immunol 15: 12-19.

Hultmark D, Engström A, Bennich H, Kapur R, Boman HG (1982) Insect immunity: Isolation and structure of cecropin D and four minor antibacterial components from Cecropia pupae. Eur J Biochem 127: 207-217.

Iwanaga S, Lee BL (2005) Recent advances in the innate immunity of invertebrate animals. J Biochem Mol Biol 38: $128-150$.

Kanost MR, Dai W, Dunn PE (1988) Peptidoglycan fragments elicit antibacterial protein synthesis in larvae of Manduca sexta. Arch Insect Biochem Physiol 8: 147-164.

Kurata S (2004) Recognition of infectious non-self and activation of immune responses by peptidoglycan recognition protein (PGRP) - family members in Drosophila. Dev Comp Immunol 28: 89-95.

Kurata S, Ariki S, Kawabata S-J (2006) Recognition of pathogens and activation of immune responses in Drosophila and horseshoe crab innate immunity. Immunobiology 211: $237-249$.

Laemmli UK (1970) Cleavage of structural proteins during the assembly of the head of bacteriophage T4. Nature 277: 680-685.

Lavine MD, Chen G, Strand MR (2005) Immune challenge differentially affects transcript abundance of three antimicrobial peptides in hemocytes from the moth Pseudoplusia includens. Insect Biochem Mol Biol 35: 1335-1346.

Leclerc V, Reichhart J-M (2004) The immune response of Drosophila melanogaster. Immunol Rev 198: 59-71.

Leulier F, Parquet C, Pili-Floury S, Ryn JH, Caroff M, Lee WJ, Mengin-Lecreulx D, Lemaitre B (2003) The Drosophila immune system detects bacteria through specific peptidoglycan recognition. Nat Immunol 4: 478-484.

Lowenberger C (2001) Innate immune response of Aedes aegypti. Insect Biochem Mol Biol 31: 219-229.
Mak P, Chmiel D, Gacek GJ (2001) Antibacterial peptides of the moth Galleria mellonella. Acta Biochim Polon 48: 1191-1195.

Marin D, Dunphy GB, Mandato CA (2005) Cyclic AMP affects the haemocyte responses of larval Galleria mellonella to selected antigens. J Insect Physiol 51: 575-586.

Morishima I, Horiba T, Iketani M, Nishioka E, Yamano Y (1995) Parallel induction of cecropin and lysozyme in larvae of the silkworm Bombyx mori. Dev Comp Immunol 19: 357-363.

Norris JL, Manley JL (1992) Selective nuclear transport of the Drosophila morphogen dorsal can be established by a signaling pathway involving the transmembrane protein Toll and protein kinase A. Genes Dev 6: 1654-1667.

Pinheiro VB, Ellar DJ (2006) How to kill a mocking bug? Cell Microbiol 8: 545-557.

Powning RF, Davidson WJ (1973) Studies on insect bacteriolytic enzymes. I. Lysozyme in haemolymph of Galleria mellonella and Bombyx mori. Comp Biochem Physiol B 45: 669-686.

Royet J, Reichhart J-M, Hoffmann JA (2005) Sensing and signalling during infection in Drosophila. Curr Opin Immunol 17: 11-17.

Schägger H, von Jagow G (1987) Tricine-sodium dodecyl sulfate-polyacrylamide gel electrophoresis for the separation of proteins in the range from 1 to $100 \mathrm{kDa}$. Anal Biochem 166: 368-379.

Schoofs L, Holman GM, Hayes TK, Nachman RJ, De Loof A (1990) Locusta tachykinin I and II, two novel insect neuropeptides with homology to peptides from the vertebrate tachykinin family. FEBS Lett 261: 397-401.

Shimabukuro M, Xu J, Sugiyama M, Taniai K, KadonoOkuda K, Kato Y, Yamamoto M, Chowdhury S, Choi SK, Choi HK, Miyanoshita A, Debnath NC, Yamakawa M (1996) Signal transduction for cecropin B gene expression in adherent hemocytes of the silkworm, Bombyx mori (Lepidoptera: Bombycidae). Appl Entomol Zool 31: 135-143.

Sun S-C, Asling B, Faye I (1991) Organization and expression of the immunoresponsive lysozyme gene in the giant silk moth, Hyalophora cecropia. J Biol Chem 266: 6644-6649.

Taniai K, Furukawa S, Shono T, Yamakawa M (1996) Elicitors triggering the simultaneous gene expression of antibacterial proteins of the silkworm, Bombyx mori. Biochem Biophys Res Commun 226: 783-790.

Tanji T, Ip YT (2005) Regulators of the Toll and Imd pathways in the Drosophila innate immune response. Trends Immunol 26: 193-198.

Vilcinskas A, Matha V (1997) Effect of the entomopathogenic fungus Beauveria bassiana on the humoral immune response of Galleria mellonella larvae (Lepidoptera: Pyralidae). Eur J Entomol 94: 461-472.

Wang L, Ligoxygakis P (2006) Pathogen recognition and signalling in the Drosophila innate immune response. Immunobiology 211: 251-261.

Wojda I, Kowalski P, Jakubowicz T (2004) JNK MAP kinase is involved in the humoral immune response of the greater wax moth larvae Galleria mellonella. Arch Insect Biochem Physiol 56: 143-154.

Yamakawa M, Tanaka H (1999) Immune proteins and their gene expression in the silkworm, Bombyx mori. Dev Comp Immunol 23: 281-289.

Zakarian RJ, Dunphy GB, Rau ME, Albert PJ (2003) Kinases, intracellular calcium, and apolipophorin-III influence the adhesion of larval hemocytes of the lepidopterous insect, Galleria mellonella. Arch Insect Biochem Physiol 53: 158-171. 